\title{
Power Converter Maintenance Optimization Using a Model-Based Digital Reliability Twin Paradigm
}

\author{
$1^{\text {st }}$ Lukas Felsberger \\ Technology Department/MNM-Team \\ CERN/Ludwig-Maximilians-Universität München \\ Genève, Switzerland/München, Germany \\ lukas.felsberger@cern.ch
}

\author{
$2^{\text {nd }}$ Benjamin Todd \\ Technology Department \\ CERN \\ Genève, Switzerland \\ btodd@cern.ch
}

\author{
$3^{\text {rd }}$ Dieter Kranzlmüller \\ MNM-Team \\ Ludwig-Maximilians-Universität München \\ München, Germany \\ kranzlmueller@ifi.lmu.de
}

\begin{abstract}
Optimization of operations and maintenance activities in factories was estimated to have a global economic potential of 1.2 to 3.7 trillion USD by recent studies. Digital twins offer a framework to achieve such optimization by studying potential improvements in the virtual space before applying them to the real world. We studied the use of a digital twin based on a general model of system failure behaviour for maintenance optimization by combining existing methodologies into a general framework. Applying it to a real-world power converter use case, we identified either reactive or preventive maintenance to be more cost-effective depending on the operating conditions. This allowed to predict optimal maintenance for existing and future systems.
\end{abstract}

Index Terms-Preventive Maintenance, Physics-of-Failure, Digital Twin, Virtual Factory

\section{INTRODUCTION}

Optimization of operations and maintenance activities in factories was estimated to have a global economic potential of 1.2 to 3.7 trillion USD by recent studies [1]. Digital twins offer a framework to achieve such optimization by studying potential improvements in the virtual space before applying them to the real world. We studied the use of a digital twin based on a general model of system failure behaviour for maintenance optimization by combining existing methodologies into a general framework and applying it to a real-world use case.

\section{Related Work}

Accurate models of failure behaviour are essential for any successful operational optimization. Such models can generally be obtained in a data-driven fashion or with a modelbased or Physics-of-Failure (PoF, [2]) approach ${ }^{1}$. Data-driven approaches are characterized by large available data-sets and limited required domain knowledge. Often, data driven models

This work has been sponsored by the German Federal Ministry of Education and Research (grant no. 05E12CHA). The final publication is available at IEEEexplore via https://doi.org/10.1109/ICSRS48664.2019.8987629 and can be cited as "Felsberger, Lukas, Benjamin Todd, and Dieter Kranzlmüller "Power Converter Maintenance Optimization Using a Model-Based Digital Reliability Twin Paradigm." 2019 4th International Conference on System Reliability and Safety (ICSRS). IEEE, 2019.”

${ }^{1}$ Common approaches often mix physics and data-driven approaches.

978-1-7281-4781-9/19/\$31.00 @2019 IEEE cannot be transferred to applications with different operating conditions than those the model was inferred from [3]. PoF approaches require a physical understanding of the investigated failure mechanisms. They allow to transfer models across operating conditions if the physics behind a failure mechanism is understood. However, in many cases the details of failure mechanisms remain unclear.

Generally, precise models requiring little data for parameter inference are desirable as obtaining data on system reliability often implies expensive destructive experimentation, competitive disadvantages due to an increased time-to-market, or costly interruptions of operations. Ideally, a framework should allow an intuitive integration of heterogeneous pieces of evidence into a single consistent model. The physics-of-failure approach comes close to such a requirement as, based on a detailed understanding and quantification of the underlying failure physics, it allows to incorporate additional data from different sources systematically.

Reliability data is often scarce and collected under different operating conditions (e.g. data from a specific car model in field use around the globe). Monitoring of operating conditions and failure behaviour is possible with modern sensor and data transmission technology. Combining data on operating conditions with failure data allows to determine operation condition dependent degradation models, here called digital reliability twins, using parameter inference strategies pioneered in the field of accelerated life testing [4].

Once such a parametric reliability model has been inferred, it can be combined with a simulation engine (see e.g. [5], [6]) to study various operational strategies and optimize realworld systems. To choose an optimal strategy, the simulation engine needs to be combined with a cost model which defines an optimization objective. Various cost models have been introduced in the literature [7].

A digital twin for reliability optimization integrates all these approaches into a single framework. It can be used to optimize operations of existing and future systems under various operating conditions, as illustrated in figure 1. It has been discussed conceptually in the literature ( [8], [9]) and first application examples have been presented ( [3], [10]-[12]), mainly for aeronautics. Our objective was to add to the discussion a general methodology for maintenance optimization using a 


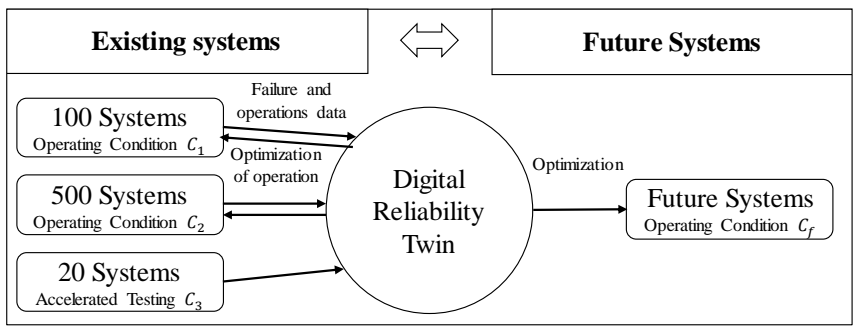

Fig. 1. Overview of the approach. Data from a system operating at different conditions is combined to form a digital reliability twin which can be used to optimize existing and future operations.

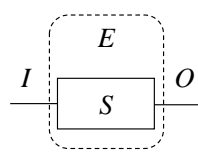

(a)

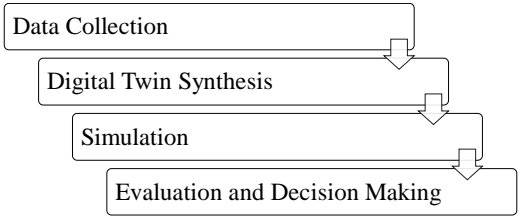

(b)
Fig. 2. (a) Illustration of a simple system $S$ with inputs $\mathbf{I}$, outputs $\mathbf{O}$ within an environment $\mathbf{E}$. (b) Overview of the methodology.

digital twin based on detailed understanding of the system failure mechanisms, an application of the outlined framework to a real-world power electronics application with a focus on failure modeling, simulation, and evaluation, and some lessons learned.

The rest of this paper is structured as follows: Section II introduces a general load-dependent model of the failure behavior which is at the heart of the digital reliability twin, a methodology to obtain a digital twin from data of existing systems, and a general simulation strategy to optimize timebased preventive maintenance. In section III, we apply the framework to an existing power converter system to evaluate the most cost-effective maintenance solution under new operating conditions and discuss results and lessons learned. Section IV concludes with a summary and a brief outlook.

\section{Methodology}

A simple system, as considered in figure $2 \mathrm{a}$, is defined to be functional if it provides the desired output $\mathbf{O}$ given that the input $\mathbf{I}$ and environment $\mathbf{E}$ are within a pre-specified range. It is faulty, if it fails to deliver the desired output $\mathbf{O}$, despite the inputs $\mathbf{I}$ and environment $\mathbf{E}$ being within a specified range (i.e. an internal failure occurred). The probability of being in a faulty state as a function of time $t$ is $F(t)$, given by $F(t)=1-\prod_{j=1}^{M}\left(1-F_{j}(t)\right)$, with $F_{j}(t)$ being independently competing failure modes (see e.g. [13]) of the system. Failure modes are commonly modeled by the two-parameter Weibull distribution, $F_{j}\left(t ; \eta_{j}, \beta_{j}\right)=1-\exp \left(-t / \eta_{j}\right)^{\beta_{j}}, t>0$, with $\eta_{j}$ being the characteristic lifetime (with the property $F_{j}(t=$ $\left.\eta_{j}\right) \approx 0.63212$ ) and $\beta_{j}$ being the shape parameter indicating an increasing $\left(\beta_{j}>1\right)$, constant $\left(\beta_{j}=1\right)$, or decreasing $\left(\beta_{j}<1\right)$ failure rate with time for failure mode $j$. [14]

A failure mode can be associated with a failure driver, $\xi_{\mathbf{j}}$, which causes degradation over time. For example, increased temperatures lead to a faster evaporation of the electrolyte in capacitors and degrade its capacity. In this case, the failure driver is the temperature and the failure mode is capacity degradation. The stress which drives degradation can be related to system operating conditions, $\mathbf{C}=[\mathbf{I}, \mathbf{O}, \mathbf{E}]$, using empiric or analytic models, $\xi_{\mathbf{j}}=\Gamma_{j}(\mathbf{C} ; \boldsymbol{\Lambda})$, with parameters $\boldsymbol{\Lambda}$. In the capacitor case, such a model would relate the temperature and the operational condition causing a temperature rise, e.g. heat dissipation due to electrical currents in nearby electronics.

Acceleration factor modeling allows to quantify the relationship between the failure driver and the probability of a failure of a unit over time. Using the two-parameter Weibull distribution, the acceleration factor is given by, $A F_{j}\left(\xi_{\mathbf{j}}, \xi_{\mathbf{j}, \mathbf{r e f}} ; \boldsymbol{\Theta}\right)=$ $\eta_{j, \text { ref }} / \eta_{j}$, with $\eta_{j, \text { ref }}$ being the characteristic lifetime at a reference operating condition ${ }^{2}, \xi_{\mathbf{j}, \text { ref }}=\Gamma_{j}\left(\mathbf{C}_{\text {ref }}\right)$, and parameters $\Theta$. Common choices for the acceleration factor model are exponential or power-law. [15]

Combining all concepts, we obtain a system failure probability,

$$
\begin{aligned}
& F\left(t, \mathbf{C} ; \eta_{j, r e f}, \beta_{j}, \boldsymbol{\Lambda}, \boldsymbol{\Theta}\right)= \\
& 1-\prod_{j=1}^{M}\left[1-\exp \left(\frac{-t \cdot A F_{j}\left(\boldsymbol{\Gamma}_{\mathbf{j}}(\mathbf{C} ; \boldsymbol{\Lambda}), \xi_{\mathbf{j}, \text { ref }} ; \boldsymbol{\Theta}\right)}{\eta_{j, r e f}}\right)^{\beta_{j}}\right]
\end{aligned}
$$

allowing to incorporate a detailed understanding of the relationship between failure mode mechanisms, failure drivers, and operating conditions. In its current formulation, the model is limited to constant-in-time loads. It can be extended to nonconstant load by using a cumulative exposure model (see e.g. [16], [17]).

\section{A. Digital Reliability Twin}

The idea of a digital reliability twin is to mimic the failure behaviour of a system under various operating conditions. Figure $2 \mathrm{~b}$ summarizes the methodology to obtain a virtual reliability twin based on model (1) from existing operation data and to use it to optimize future operations as illustrated in figure 1. In the following the methodology will be discussed in detail.

a) Data Collection: For a system $i$ the failure modes and times $t_{f, i, j}$ and operating conditions $\mathbf{C}_{\mathbf{i}}$ need to be collected ${ }^{3}$. Data on the relationship between the failure drivers $\xi_{\mathbf{j}}$ and operating conditions $\mathbf{C}$ can either be collected in-situ or in separate experiments. Note that the failure mechanism and its

\footnotetext{
${ }^{2}$ Note that we assume uniform acceleration, i.e. $\beta_{j, r e f}=\beta_{j}$. The methodology can be extended to non-uniform acceleration. However, a changing $\beta_{j}$ indicates a changing failure mode and should be divided in separate failure modes.

${ }^{3}$ The methodology does not impose restrictions on Type I/II censored or partially missing data.
} 
physics need to be quantitatively understood to determine the failure drivers to be measured.

b) Digital Twin Synthesis: Using data from $N$ identical systems operated under different conditions $\mathbf{C}_{\mathbf{i}}$, the parameters of model (1) can be inferred using methods of accelerated life testing. As failure data is usually scarce and expensive to obtain, the parameter uncertainty should be quantified and propagated to the simulation engine. E.g., MaximumLikelihood or Monte-Carlo or Particle Filter based Bayesian inference methods can be employed for parameter and uncertainty estimation. For a detailed discussion refer to [4], [18].

Generally, the validity of the models in terms of input parameter intervals shall be specified to prevent inaccurate extrapolations. E.g. learning a temperature dependent empirical model from field use at temperatures between 0 and 40 degrees Celsius is expected to be inaccurate for cryogenic environments.

c) Simulation Engine: A simulator is used to study the optimal maintenance strategy. Both the failure behaviour and the maintenance and repair actions are simulated. The failure behaviour has been characterized by model (1). The maintenance strategies studied are time-based preventive maintenance (repairing the system after a certain operational time $t_{r, p} \sim \mathcal{N}^{R}\left(T_{r, p}, \sigma_{r, p}\right)$, with $\mathcal{N}^{R}$ being a rectified Normal distribution) and reactive maintenance (repairing the system after a failure has occurred within a time $t_{r, c} \sim \mathcal{N}^{R}\left(T_{r, c}, \sigma_{r, c}\right)$ ). In both cases we assumed perfect repair (i.e. the system is as good as new after repair).

To simulate different maintenance strategies for a system operational lifetime, $T_{O L}$, a layered approach is chosen as illustrated in figure $4 \mathrm{a}$.

- Core model: At the core level, we simulate a single system stochastically for a given parametrization of model (1) and repair strategy. The state diagram of the core simulation is illustrated in figure 3 . There are two states: 'Operation' and 'Down'. The simulation starts with lifetimes $t_{f, j}$ for each of the competing failure modes drawn from model (1). If the system operates until $t_{r, p}>\min \left(t_{f, j}\right)$, without any failure occurring, a preventive repair is carried out which resets the system lifetimes $t_{f, j}$. If a failure occurs before the scheduled preventive repair, the system enters the 'Down' state and a corrective repair within $t_{r, c}$ is carried out. If the simulation time exceeds the operational lifetime, $t>T_{O L}$, the simulation is terminated and its results $\mathbf{R}_{\mathbf{k}}=\left(n_{r, k}, n_{d, k}\right), n_{r, k}$ being the number of repair actions (both preventive and reactive) executed during simulation, $n_{d, k}$ the number of transitions to the 'Down' state leading to an unexpected interruption of operations for the k-th evaluation, saved.

- Core Initializer: The stochastic core level is initialized in a Monte Carlo approach to compute the mean of the core results, $\mathbf{R}_{\text {core }}=\sum_{k=1}^{K} R_{k} / K$.

- UQ Initializer: To perform a sensitivity analysis or uncertainty quantification, this layer draws $L$ i.i.d. initialization parameters from their probability density functions and

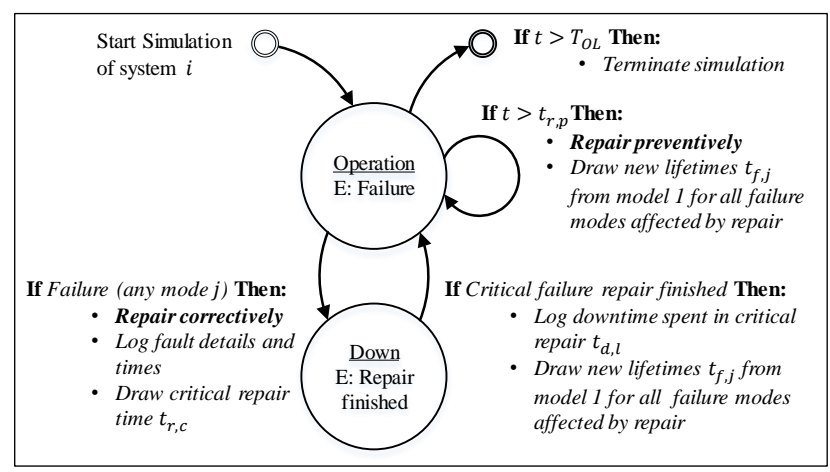

Fig. 3. State diagram and state transition conditions of Core model.

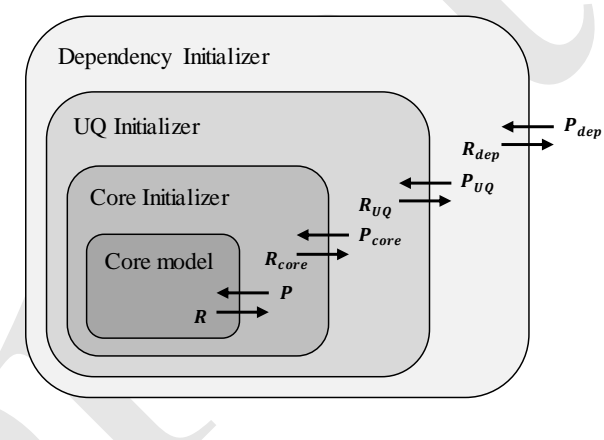

(a)

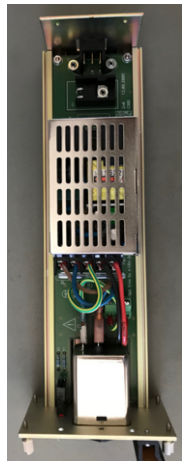

(b)
Fig. 4. (a) Layered simulation approach (adapted [17]). (b) Power converter.

passes them to the core initializer. Results from all evaluations are stored to calculate statistics.

- Dependency Initializer: This layer allows to study parameter dependencies by passing a series of defined parameter combinations to the lower layers.

d) Evaluation and Decision Making: Adding a cost function to the simulation engine allows to optimize operation by searching over a parameter grid characterizing different maintenance strategies. We define a simple model for operational cost, $C=n_{r} \cdot c_{r}+n_{d} \cdot c_{d}$, over an operational lifetime $T_{O L}$, with $n_{r}$ being the mean number of repair actions, $n_{d}$ the mean number of transitions to the 'Down' state, $c_{r}$ the average cost associated with a single repair action and $c_{d}$ being the average cost associated with an unexpected interruption of operations. $^{4}$

\section{USE-CASE}

We applied the introduced framework to optimize the maintenance of a switch-mode power supply as illustrated in figure 4b. A digital reliability twin was learned from past operation under different operating conditions. Then, it was used to optimize maintenance for scenarios with new operating conditions.

\footnotetext{
${ }^{4}$ Note that many other cost models would be possible with the chosen simulation approach.
} 
a) Data Collection and Digital Twin Synthesis: 786 power converters had been operated for more than ten years during which 59 failures with times $t_{f, i, j}$, modes $j$, and operational conditions, $\mathbf{C}_{\mathbf{i}}$, had been recorded [19]. The systems had been operated in a particle accelerator environment at controlled ambient temperatures and with $n=580,90,116$ systems at three different constant system loads of $I=$ $0.4,0.9,1.2 A$. A reliability investigation [20] had identified three failure modes. The first failure mode was fuse wear and was directly proportional to the system current $I$, most likely due to repeated heating and cooling by the alternating current [21]. The second failure mode was much less frequent and hence its physics were not explored. System current was chosen as failure driver as it was correlated. The third wear out failure was due to evaporation of the electrolyte in capacitors. An investigation of the failure mechanism revealed that the evaporation was due to heating of the capacitors. The relation between system current $I$ and capacitor temperature $T$ was obtained experimentally at ambient temperatures (see [20], [22]). The acceleration model was taken from the literature [23], as the failure mode was only observed under one operational setting and, hence, could not be inferred from the recorded failure data.

TABLE I

FAILURE MODE PARAMETERS. ADAPTED FROM [17].

\begin{tabular}{|c|c|c|c|}
\hline \multirow{2}{*}{$\begin{array}{c}\text { Para- } \\
\text { meters }\end{array}$} & \multicolumn{3}{|c|}{ Failure Modes } \\
\cline { 2 - 4 } & Fuse $\boldsymbol{j}=\mathbf{1}$ & Empirical $\boldsymbol{j}=\mathbf{2}$ & Capacitor $\boldsymbol{j}=\mathbf{3}$ \\
\hline$\xi_{j}$ & $I[A]$ & $I[A]$ & $T[K]$ \\
\hline$\Gamma_{j}$ & $I$ & $I$ & $T=55\left(1-e^{-0.7 I}\right)+298$ \\
\hline$A F_{j}$ & $\left(\frac{I}{I_{\text {ref }}}\right)^{1.0}$ & $\left(\frac{I}{I_{\text {ref }}}\right)^{0.6}$ & $\exp \left(\frac{0.94}{k_{b}}\left(\frac{1}{T_{\text {ref }}}-\frac{1}{T}\right)\right)$ \\
\hline$\eta_{j, \text { ref }}$ & $19219 d$ & $76768 d$ & $4200 d$ \\
\hline$\beta_{j, \text { ref }}$ & 1.16 & 0.9 & 8.3 \\
\hline$\xi_{\text {ref }}$ & 1.2 & 1.2 & $330 K$ \\
\hline
\end{tabular}

Using failure and operation condition data, a quantification in accordance with model (1) could be obtained by using maximum likelihood estimation [22]. The parameter estimates have been verified by reproducing the original failure behaviour in [17] and were summarized in table I. However, due to the limited amount of available failure data, the parameter estimates were considered uncertain.

b) Simulation and Results: We considered a scenario with two future operating conditions at system loads of $I=0.3,1.6 \mathrm{~A}$ and ambient temperatures. A repair is carried out by replacing the whole power converter at a repair cost $c_{r}=150 m u^{5}$ [19]. A failure leading to unplanned downtime was assumed to cost $c_{d}=1000 \mathrm{mu}$. The objective was to find the most cost effective maintenance strategy for an operational lifetime $T_{O L}=10^{4} d(d=$ days $)$. We therefore varied the preventive repair time from $T_{r, p}=10^{2} d$ to $10^{4} d$ with a fixed preventive repair interval variance $\sigma_{r, p}=30 d$. Note, that for $T_{r, p}=T_{O L}=10^{4} d$ the case of fully reactive maintenance is obtained (no scheduled preventive repairs). The duration of

${ }^{5} \mathrm{mu}$ stands for monetary unit.

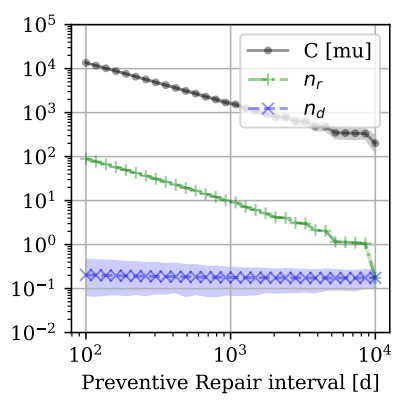

(a) $0.3 \mathrm{~A}$

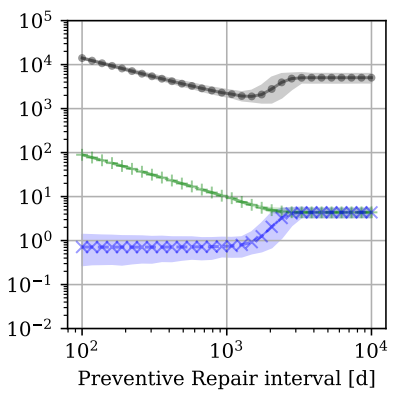

(b) $1.6 \mathrm{~A}$
Fig. 5. Number of downtime events $n_{d}$, repairs $n_{r}$ and operational cost $C$ as a function of preventive repair interval $T_{r, p}$. The shaded area depicts the $95 \%$ empirical highest probability density region, the lines the mean, and the markers the discrete evaluation points.

a corrective repair was set to zero, $T_{r, c}, \sigma_{r, c}=0$, as it is irrelevant for the chosen cost model.

Since the parameter uncertainty had not been estimated in [20] and [22], we carried out a sensitivity analysis by comparing the results when using deterministic and stochastic input parameters; the latter drawn from a Normal distribution with means given by the deterministic parameter values (table I) and variances of $10 \%$ of their mean.

The results of the simulations with stochastic failure mode parameters from table I, repair parameters $T_{r, p}$ and $\sigma_{r, p}$, a system load of $I=0.3,1.6 \mathrm{~A}$, and ambient environment temperatures are shown in figure $5 \mathrm{a}$ and $5 \mathrm{~b}$, respectively. ${ }^{6}$ For $0.3 A$, the operational cost $C$ was the lowest for the case of fully reactive maintenance, $T_{r, p}=T_{O L}=10^{4} d$, for which the number of repairs, $n_{r}$, equaled the number of downtime events $n_{d}$ (i.e. no system was changed preventively). For 1.6A, the operation cost $C$ was the lowest for a preventive repair interval of $T_{r, p} \approx 1500 d$. For $T_{r, p}>3000 d$, the maintenance was effectively reactive as the number of repairs equaled the number of downtime events. For $T_{r, p}<1000 d$, the number of repairs increased without reducing the number of downtime events.

c) Discussion: Comparing the plots for $0.3 \mathrm{~A}$ and $1.6 \mathrm{~A}$ showed very different results for the most cost effective maintenance approach. For low system currents $(I<0.4)$, reactive maintenance was the most cost efficient solution, whereas for higher system currents $(I>0.7)$ preventive maintenance was more cost efficient. This behaviour was summarized in table II which shows the minimal operational cost and corresponding optimal maintenance strategy for different system currents. The table also compares results obtained with deterministic failure parameters (D) and stochastic failure parameters (S) (with a variance of $10 \%$ of their mean). The estimated optimal

\footnotetext{
${ }^{6}$ We drew $L=200$ parameter combinations from a Normal distribution with means given by the deterministic parameter values (table I) and variances of $10 \%$ of their mean and used $K=10^{4}$ Monte-Carlo samples to evaluate $\mathbf{R}_{\text {core }}$ for each parameter combination to obtain these results. Using $K=$ $10^{4}$ MC samples resulted in a relative standard deviation of the mean of $C$ of less than $3 \%$ over repeated runs with deterministic parameterization.
} 
maintenance strategy and the mean of the operational cost $C$ were minimally affected by the uncertainty in the input parameters. For system currents $I>0.7 \mathrm{~A}$, preventive maintenance led to a lower operational cost than reactive maintenance $\left(T_{r, p} \geq 10^{4}\right)$.

TABLE II

OPTIMAL REPLACEMENT TIME $T_{r, p}$ AND CORRESPONDING MEAN COST $C$ (AND STDDEV.) AS FUNCTION OF SYSTEM CURRENT $I$. (S) DEPICTS RESULTS OBTAINED USING UNCERTAIN FAILURE PARAMETERIZATION AND (D) FOR USING DETERMINISTIC FAILURE PARAMETERIZATION.

\begin{tabular}{|c|c|c|c|c|}
\hline Curr. [A] & $\mathbf{T}_{\mathbf{r}, \mathbf{p}}[d](\boldsymbol{S})$ & $\boldsymbol{C}[\mathbf{m u}](\boldsymbol{S})$ & $\mathbf{T}_{\mathbf{r}, \mathbf{p}}[d](\boldsymbol{D})$ & $\boldsymbol{C}[\mathbf{m u}](\boldsymbol{D})$ \\
\hline 0.19 & $10^{4}$ & $139 \pm 34$ & $10^{4}$ & $131 \pm 3$ \\
\hline 0.38 & $10^{4}$ & $248 \pm 50$ & $10^{4}$ & $240 \pm 5$ \\
\hline 0.74 & 5300 & $590 \pm 86$ & 6210 & $572 \pm 6$ \\
\hline 1.45 & 1740 & $1624 \pm 231$ & 1740 & $1543 \pm 8$ \\
\hline
\end{tabular}

A closer look on the failure modes revealed an explanation why preventive maintenance did not reduce the number of downtime events for low system loads. Failure modes 1 and 2 show no wear-out behaviour but are rather constant in time $(\beta \approx 1)$, whereas failure mode 3 shows a strong wear-out behaviour $(\beta=8.3)$. However, the characteristic lifetime of $\eta_{3}$ exceeds the operational lifetime of the system $T_{O L}$ for system loads $I<0.7 A$. Hence, effectively the system had no wear-out behaviour for low system loads and preventive replacements do not reduce downtime events as new systems are as likely to fail as old ones. This showed that detailed understanding and quantification of the failure modes is necessary to optimize maintenance strategies for new operating conditions.

d) Lessons Learned: Based on the experience from the outlined use-case, following recommendations could be given:

- Despite a large fleet of 786 systems and a decade of operational experience less than 60 failures distributed over three different failure modes have been observed. Therefore, it is imperative to use 'data-efficient' approaches, such as Physics-of-Failure, to quantify the failure modes.

- Compared to the time, knowledge and effort required for data collection and failure mode quantification, computational requirements for running Monte-Carlo simulations of digital reliability twins seemed less taxing.

\section{Summary, Conclusions and OUtlook}

We presented a general methodology for maintenance optimization using a digital twin. Based on failure mechanism understanding and inference strategies from accelerated life testing, a operation condition dependent system failure behaviour can be quantified. Such a model can be used to simulate different operation conditions and maintenance strategies. Combined with a cost model, the optimal maintenance strategy can be found for a certain operating condition of potential future systems. Applying the framework to optimize power converter maintenance, we identified either reactive or preventive maintenance to be more cost-effective depending on the system current.

For future work, the methodology could be verified with more use-cases and extended to time-varying system loads for a wider applicability. It can be combined with more complex cost models, including time-to market and development costs, to study e.g. the cost effectiveness of additional accelerated tests during development.

\section{ACKNOWLEDGMENT}

We would like to thank T. C.-Michaud and A. C.N. Oliveira for helpful inputs and discussions and the CERN-Technology department for providing their data and resources.

\section{REFERENCES}

[1] J. Manyika, M. Chui, P. Bisson, J. Woetzel, R. Dobbs, J. Bughin, and D. Aharon, "Unlocking the potential of the internet of things," McKinsey Global Institute, 2015.

[2] K. Chatterjee, M. Modarres, and J. B. Bernstein, "Fifty years of physics of failure," J. Rel. Inf. Anal. Center, vol. 20, no. 1, pp. 1-5, 2012.

[3] F. Tao, J. Cheng, Q. Qi, M. Zhang, H. Zhang, and F. Sui, "Digital twindriven product design, manufacturing and service with big data," The International Journal of Advanced Manufacturing Technology, vol. 94 no. 9-12, pp. 3563-3576, 2018.

[4] W. B. Nelson, Accelerated testing: statistical models, test plans, and data analysis. John Wiley \& Sons, 2009, vol. 344.

[5] P. Hall and J. Strutt, "Probabilistic physics-of-failure models for component reliabilities using monte carlo simulation and weibull analysis: a parametric study," Reliability Engineering \& System Safety, vol. 80, no. 3, pp. 233-242, 2003.

[6] F. Long, P. Zeiler, and B. Bertsche, "Modelling the production systems in industry 4.0 and their availability with high-level petri nets," IFACPapersOnLine, vol. 49, no. 12, pp. 145-150, 2016.

[7] S. Alaswad and Y. Xiang, "A review on condition-based maintenance optimization models for stochastically deteriorating system," Reliability Engineering \& System Safety, vol. 157, pp. 54-63, 2017.

[8] E. Glaessgen and D. Stargel, "The digital twin paradigm for future nasa and us air force vehicles," in 53rd AIAA/ASME/ASCE/AHS/ASC Structures, Structural Dynamics and Materials Conference 20th AIAA/ASME/AHS Adaptive Structures Conference 14th AIAA, 2012, p. 1818.

[9] T. Gabor, L. Belzner, M. Kiermeier, M. T. Beck, and A. Neitz, "A simulation-based architecture for smart cyber-physical systems," in 2016 IEEE International Conference on Autonomic Computing (ICAC). IEEE, 2016, pp. 374-379.

[10] K. Reifsnider and P. Majumdar, "Multiphysics stimulated simulation digital twin methods for fleet management," in 54th AIAA/ASME/ASCE/AHS/ASC Structures, Structural Dynamics, and Materials Conference, 2013, p. 1578.

[11] E. J. Tuegel, A. R. Ingraffea, T. G. Eason, and S. M. Spottswood, "Reengineering aircraft structural life prediction using a digital twin," International Journal of Aerospace Engineering, vol. 2011, 2011.

[12] A. Cerrone, J. Hochhalter, G. Heber, and A. Ingraffea, "On the effects of modeling as-manufactured geometry: toward digital twin," International Journal of Aerospace Engineering, vol. 2014, 2014.

[13] M. Moeschberger, "Competing risks," in Statistical Theory and Applications. Springer, 1996, pp. 279-292.

[14] W. Weibull, "A statistical distribution function of wide applicability," Journal of applied mechanics, vol. 103, no. 730, pp. 293-297, 1951.

[15] J. W. McPherson, Reliability physics and engineering. Springer, 2010.

[16] W. Nelson, "Accelerated life testing-step-stress models and data analyses," IEEE transactions on reliability, vol. 29, no. 2, pp. 103-108, 1980.

[17] L. Felsberger, B. Todd, and D. Kranzlmüller, "Cost and availability improvements for fault-tolerant systems through optimal load-sharing policies," Procedia Computer Science, vol. 151, pp. 592-599, 2019.

[18] W. B. Nelson, "A bibliography of accelerated test plans," IEEE Transactions on Reliability, vol. 54, no. 2, pp. 194-197, 2005.

[19] C. Martin, "Cibd power supply overview," https://cernbox.cern.ch/index.php/s/SqjbaMTdrqObcql, 2018, Presented at the Reliability and Availability Studies Working Group meeting, CERN, Geneva, [Online; accessed 20-August-2019].

[20] C. Martin, B. Todd, and Y. Thurel, "Hccibd reliability analysis," https://cernbox.cern.ch/index.php/s/10hycRrGn4aCNVY, 2018, Presented at the Reliability and Availability Studies Working Group meeting, CERN, Geneva, [Online; accessed 26-June-2019]. 
[21] X. Meng and J. Sloot, "Reliability concept for electric fuses," IEEE Proceedings-Science, Measurement and Technology, vol. 144, no. 2, pp. 87-92, 1997.

[22] L. Felsberger, "Combining reliability statistics with physics of failure to study and simulate the operational availability of redundant power converters," https://cernbox.cern.ch/index.php/s/W97xCUG3926Ne0a, 2018, Presented at the Reliability and Availability Studies Working Group meeting, CERN, Geneva, [Online; accessed 26-June-2019].

[23] S. G. Parler Jr and P. C. Dubilier, "Deriving life multipliers for electrolytic capacitors," IEEE Power Electronics Society Newsletter, vol. 16, no. 1, pp. 11-12, 2004. 